\title{
Search for Fractional-Charge Particles in Meteoritic Material
}

\author{
Peter C. Kim, Eric R. Lee, Irwin T. Lee, and Martin L. Perl* \\ Stanford Linear Accelerator Center, Stanford University, 2575 Sand Hill Road, Menlo Park, California 94025, USA \\ Valerie Halyo \\ Department of Physics, Princeton University, Princeton, New Jersey 08544, USA \\ Dinesh Loomba \\ Department of Physics, University of New Mexico, Albuquerque, New Mexico 87131, USA*
}

\begin{abstract}
We have used an automated Millikan oil drop method to search for free fractional-charge particles in a sample containing in total $3.9 \mathrm{mg}$ of pulverized Allende meteorite suspended in $259 \mathrm{mg}$ of mineral oil. The average diameter of the drops was $26.5 \mu \mathrm{m}$ with the charge on about 42500000 drops being measured. This search was motivated by the speculation that isolatable, fractional-charge particles produced in the early Universe and present in our Solar System are more likely to be accumulated in asteroids than on Earth's surface. No evidence for fractional-charge particles was found. With 95\% confidence, the concentration of particles with fractional-charge more than $0.25 e$ ( $e$ being the magnitude of the electron charge) from the nearest integer charge is less than $1.3 \times 10^{-21}$ particles per nucleon in the meteoritic material and less than $1.9 \times 10^{-23}$ particles per nucleon in the mineral oil.
\end{abstract}

Since the electron's identification and the measurement of its charge [1] $e$ about 100 years ago, there has been the question as to whether there are other isolatable elementary particles with fractional charge such as, for example, $0.5 \times$ $e$ or $\pi \times e$. No known physics law requires the unit of elementary particle charge to be unique; hence, the existence of fractional-charge, isolatable, elementary particles is an experimental question. Of course the quarks are taken to have fractional charge $\pm 2 / 3$ or $\pm 1 / 3$, but they are not isolatable.

Since about 1950, there have been many searches for fractional-charge, isolatable, elementary particles using a variety of search methods: detection in cosmic rays, production in accelerators, production in colliding beams machines, searches in bulk matter [2-14]. All but two searches reported null results. McCusker claimed detection of free quarks in early cosmic rays searches [12], but this has not been confirmed in much more extensive modern searches. Using a superconducting levitometer method, LaRue et al. [11] reported the existence of fractionalcharged particles in niobium, but Smith et al. [13] found no such particles using a ferromagnetic levitometer on a 5 times larger niobium sample.

Two searches for fractional-charge particles in meteoritic material have been carried out: the 1989 ferromagnetic levitometer search by Jones et al. [14] and the search reported in this Letter. Jones et al. searched through three samples of meteoritic material - Hoba iron-nickel, Forsyth County iron-nickel, and Murchison stoney - with a total mass of $2.8 \mathrm{mg}$. No fractional-charge particles were found.

If isolatable, fractional-charge particles exist in nature, then there should be some relic abundance left over from the early Universe. Where they would be located on Earth depends strongly on their chemical properties $[15,16]$ which require assumptions about their charge and mass. Geochemical and geophysical processes occurring during Earth's early history generally produced differentiation by mass, with heavier atoms generally tending to sink deep inside Earth. Null results from accelerator and collider searches for fractional-charge particles indicate that they are likely to be heavy; hence, they may have sunk deep into the Earth during its early formation history. However, there are important exceptions to differentiation by density; for example, minerals rich in uranium, plutonium, and lead have been pushed back to the surface of Earth by geochemical processes at the crust-mantle boundary. We have wanted our search to be as model independent as possible, and therefore these considerations have motivated us to target our search to the carbonaceous chondritic meteorites, which are some of the most primordial and unprocessed sources of materials inside the solar system. At over 4.5 Gyrs old, these meteorites are some of the oldest dated objects in the solar system and, indeed, are used to date the solar system. It is also believed that they have undergone very little processing since formation; therefore, little differentiation within the meteoritic material has occurred. Other than the loss of volatile elements, the initial relative elemental abundances have been maintained in these objects. Thus, any fractional-charge particles initially bound in these meteorites should be there at roughly the same abundances today. Finally, their elemental abundances are believed to be representative of the material from which the solar system collapsed. That is, data from these meteorites together with that from the Sun's photosphere are 
used to specify the initial elemental abundances of the solar system. If fractional charge particles exist in nature, these objects are likely to contain them.

In our search, we used a commercially purchased sample from the Allende carbonaceous chondrite meteorite [17]. The meteorite was first ground by hand and then further pulverized using a jet pulverizer, giving a range of powder sizes from 0.04 to $6.0 \mu \mathrm{m}$. It is an art, somewhat hit and miss, to make a colloidal suspension using a very diverse mineral powder. After much experimentation, using the Edison method [18], we found we could suspend $1.5 \%$ by weight powder in a mixture of $5 \%$ by volume of Castrol brand 10W30 weight motor oil and $95 \%$ by volume of Ultraol $50 \mathrm{NF}$ [19]. We were not able to significantly increase the proportion of meteorite in the suspension.

Our development of an automated Millikan oil drop method to search for free fractional-charge particles began in 1994 [20] with some of the inspiration for a modern Millikan oil drop apparatus coming form the earlier work of Bland and his colleagues at San Francisco State University $[21,22]$.

The principle of our measurement of drop charge is that a drop of radius $r$, density $\rho$, and charge $Q$ falls in air ( $z$ direction) through a horizontal ( $x$ direction) electric field of strength $E$. From Stokes" law, the horizontal terminal velocity, $v_{x}$, is

$$
v_{x}=\frac{Q E}{6 \pi \eta r}
$$

where $\eta$ is the viscosity of air. Hence, measuring $v_{x}$ gives $Q$ providing $r$ is known. The drop radius is determined from the $v_{x}$ of integer charge particles. The measurement of $Q$ does not depend on the density of the drop and is also independent of the gravitational force on the drop. The electric field alternates in the $+x$ and $-x$ direction so that the drop is moved back and forth along the $x$ axis. If the drop were falling in still air, the vertical terminal velocity would be given by

$$
v_{z, \text { term }}=\frac{2 r^{2} \rho g}{9 \eta}
$$

where $g$ is the acceleration of gravity. However, we use an upward flow of air of velocity $v_{\text {air }}$ in the $-z$ direction. Hence, the net downward velocity of the drop is

$$
v_{z}=\frac{2 r^{2} \rho g}{9 \eta}-v_{\text {air }} .
$$

We want $v_{z}$ to be small; hence, we set $v_{\text {air }}$ to be close to $v_{z, \text { term }}$ but slightly smaller.

The apparatus for this search, shown schematically in Fig. 1, consisted of the Lee et al. [23] apparatus we used previously, augmented (a) by a device, called a neutralizer, $[24,25]$ that brings the average drop charge to zero and (b) by increased automatic computer control of the drop trajectories. The piezoelectrically driven, drop-on-demand

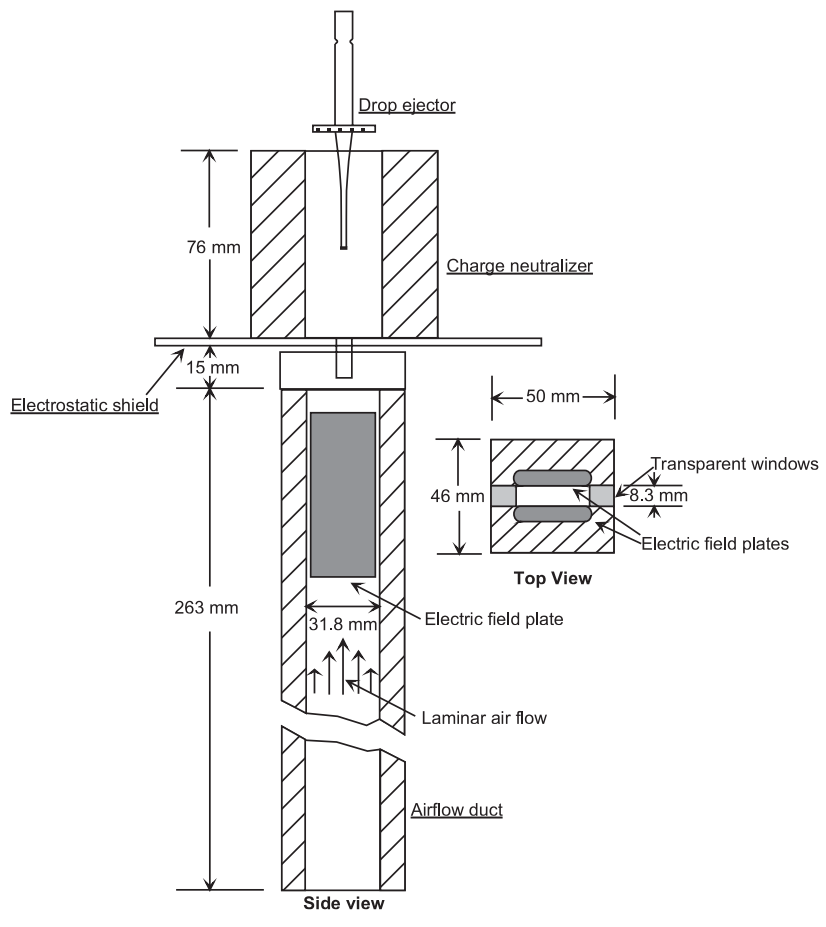

FIG. 1. Search apparatus.

drop generator [26] produced 0.833 drops per second with an average drop diameter of $26.5 \mu \mathrm{m}$ and a short term (one hour) diameter spread of $0.016 \mu \mathrm{m}(0.06 \%)$. The data were analyzed in $1 \mathrm{~h}$ segments, so a gradual long-term drift in the average drop diameter, which did in fact occur, was acceptable so long as the drop diameter was stable over durations of the order of hours.

The drops emerging from the generator had charges spread over \pm several thousand $e$ [24]. However, the precision measurement of drop charge required a very much smaller charge spread centered at zero charge. The neutralizer we built [25] consisted of a still air vertical space through which the drops fell, the air being continuously ionized by a $200 \mu \mathrm{C}{ }^{90} \mathrm{Sr}$ source. A horizontal electric field is produced across the vertical space by metal plates, the total voltage, $V$, can be varied from +3 to -3 volts. By adjusting $V$, we obtained a Gaussian drop charge distribution centered close to zero with an rms of about $15 e$. The drops leaving the neutralizer fall directly into the charge measurement chamber.

As a drop falls though the measurement chamber, there are $n_{\text {images }}$ independent measurements of its charge, $n_{\text {imagesave }}=8$. The motion of the drops in the measurement chamber is recorded using a CCD through an $f / 11$, $135 \mathrm{~mm}$ focal length lens. The image of the drops is produced by a $10 \mathrm{~Hz}$, stroboscopic light source made of 20 red LEDs. The CCD pixel images of the drops is computer processed and stored on a hard disk as described in Lee et al. [23].

Since there is a slow drift in the diameter of the ejected drops between drop ejector servicing due to debris build up 
in the ejection aperture, drop charge measurements were grouped in intervals of $1 \mathrm{~h}$ each for purposes of analysis and initial statistical cuts. Within each $1 \mathrm{~h}$ analysis interval, drop diameter consistency, trajectory, mean charge and charge spread must be within preset intervals, or the hour run is rejected. Within each good $1 \mathrm{~h}$ interval, each individual fluid drop must pass cut criteria based on consistency of multiple charge measurements of the same drop, mean drop charge, and physical location of the drop within the imaging field.

In addition to storing the data for offline processing, the computer system measures in real time the drop charge distribution and keeps the distribution centered by varying the voltage in the neutralizer. The computer system also determines in real time the average drop trajectory in the measurement chamber and corrects the trajectory, if necessary, by moving the drop generator horizontally.

The search began on January 15, 2004, and ended on December 31, 2006, with $42.5 \times 10^{6}$ drops produced which passed all measurement cuts. In a search for rare particles, the experimenter must avoid the bias that occurs when the full data set is examined, and then the data selection criteria are changed so that a statistical fluctuation becomes significant. We avoided this bias by substantially setting the drop selection criteria using early data and then looking at newly accumulated data every several months to see that the apparatus was running properly. The percentage of cut events is ill defined since a malfunctioning drop generator can skip or eject multiple off axis drops with randomly determined detectability. Using the criteria of $100 \%$ up time at an ejection rate of 0.833 drops per second, the experimental apparatus could hypothetically have been able to measure the charges on $77.8 \times 10^{6}$ drops in the absence of any measurement cuts. Combining both hardware downtime and measurement cuts, the charges of $42.5 \times 10^{6}$ drops were measured in the actual experiment.

After the application of these criteria, we had a final data sample of 42537104 drops of average diameter $26.5 \mu \mathrm{m}$. Figure 2 shows the charge distribution in units of $e$. We see sharp peaks at integer numbers of charges and no drops further than $0.25 e$ from the nearest integer. We emphasize that there is no background subtraction here; this is all the data after the application of the criteria previously discussed.

To show the shape of the peaks at integer values of $q$, we superimpose them in Fig. 3 using the charge distribution, $q_{c}$, defined by $q_{c}=q-N_{c}$ where $N_{c}$ is the signed integer closest to $q$. The peaks have a near Gaussian distribution with a standard deviation of $0.042 e$.

In Fig. 4, we superimpose the valleys between the peaks using the residual charge distribution, $q_{r}$, defined by $q_{r}=$ $|q|-N_{l}$ where $N_{l}$ is the largest integer less than $|q|$. We did not find any drops with residual charge between $0.25 e$ and $0.75 e$.

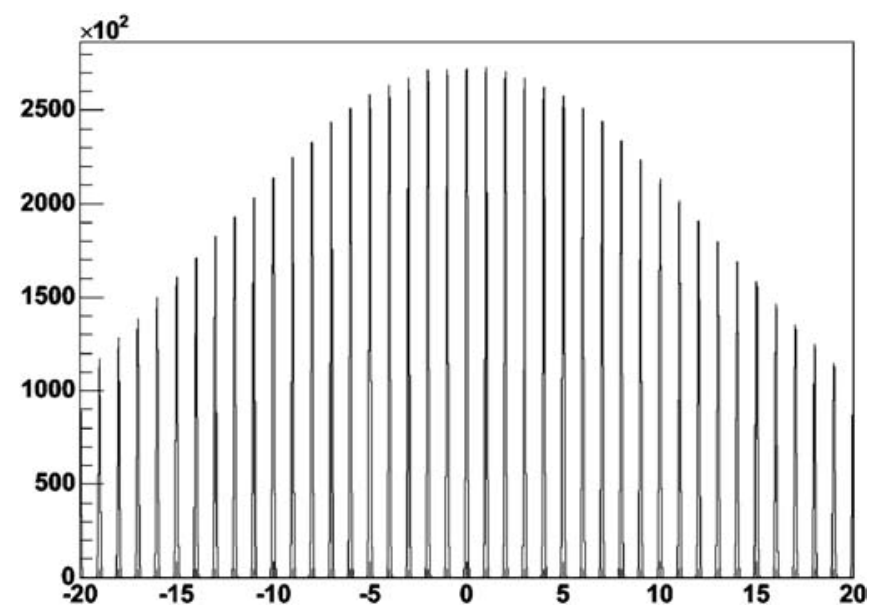

FIG. 2. The $q$ charge distribution in units of $e$.

In conclusion, with $95 \%$ confidence, the concentration of particles with fractional-charge more than $0.25 e$ from the nearest integer charge is less than $1.3 \times 10^{-21}$ particles per nucleon in the meteoritic material and less than $1.9 \times$ $10^{-23}$ particles per nucleon in the mineral oil. The total mass of our meteoritic material is $3.9 \mathrm{mg}$. Thus, our null results agree with that of Jones et al. [14], and the two searches have covered a variety of meteoritic materials with a total mass of $5.7 \mathrm{mg}$. The $259 \mathrm{mg}$ of mineral oil is the largest, fractional-charge search sample used in the Millikan oil drop method or the magnetic levitometer method.

We do not plan to continue the search for fractionalcharge particles using our drop method because the next search should use samples a hundred times larger, but we have not found a practical method to accomplish this dream. We have considered the expensive possibility of building and simultaneously operating a hundred copies of our present apparatus, but we have not solved the problem of insuring that all the machines are operating correctly. We think the next step in meteorite searches should be a

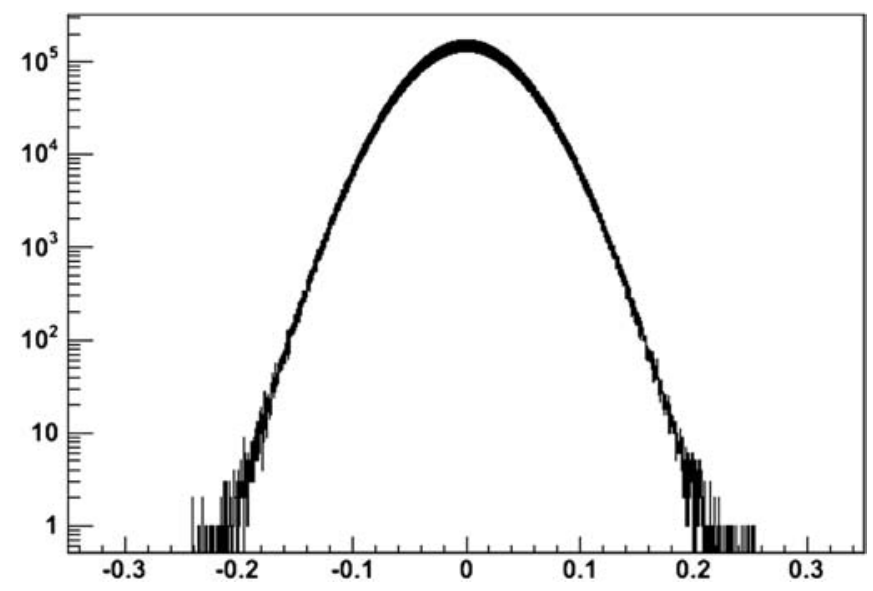

FIG. 3. The $q_{c}$ charge distribution in units of $e$. 


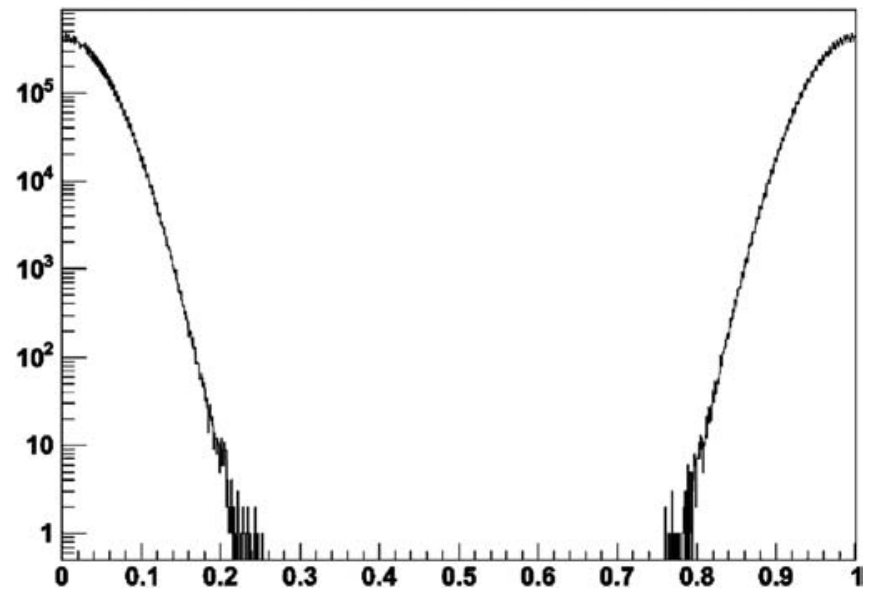

FIG. 4. The $q_{c}$ residual charge distribution in units of $e$.

ferromagnetic levitometer using full computer system automatic data acquisition and control. At present, we do not have the time or resources to carry out this proposal.

We are very grateful to our collaborators in previous fractional-charge searches: Charles Hendricks, Klaus Lackner, Nancy Mar, Howard Rogers, and Gordon Shaw; and to student programmers Adie Jain and Jason Seidman. This work was supported by Contract No. DE-AC0276 SF00515 with the U. S. Department of Energy.

*martin@slac.stanford.edu

[1] R. A. Millikan, Phys. Rev. 32, 349 (1911).

[2] L. W. Jones, Rev. Mod. Phys. 49, 717 (1977).

[3] M. Marinelli and G. Morpurgo, Phys. Rep. 85, 161 (1982).

[4] L. Lyons, Phys. Rep. 129, 225 (1985).

[5] P. F. Smith, Annu. Rev. Nucl. Part. Sci. 39, 73 (1989).
[6] M. L. Perl and E. R. Lee, Am. J. Phys. 65, 698 (1997).

[7] M. L. Perl et al., Int. J. Mod. Phys. A 16, 2137 (2001).

[8] M. L. Perl et al., Int. J. Mod. Phys. A 19, 2595 (2004).

[9] M. Marinelli and G. Morpurgo, Phys. Lett. B 137, 439 (1984).

[10] G. Morpugo et al., Nucl. Instrum. Methods 79, 95 (1970).

[11] G. S. LaRue et al., Phys. Rev. Lett. 46, 967 (1981).

[12] B. McCusker, The Quest for Quarks (Cambridge University Press, Cambridge, 1983).

[13] P. F. Smith et al., Phys. Lett. B 171, 129 (1986); P. F. Smith et al., Phys. Lett. B 181, 407 (1986); P. F. Smith et al., Phys. Lett. B 153, 188 (1985).

[14] W. G. Jones et al., Z. Phys. C 43, 349 (1989).

[15] K. S. Lackner and G. Zweig, Phys. Rev. D 28, 1671 (1983).

[16] K. S. Lackner and G. Zweig, Novel Results in Particle Physics (Vanderbilt, 1982), edited by R.S. Panvini, S. Alam, and S. E. Csorna (AIP, New York, 1982), p. 1.

[17] The Allende meteorite, weighing at least two tons, fell in Mexico in 1969, leaving fragments over a 60 square mile area.

[18] We tried a large variety of organic and inorganic liquids, mixed with all sorts of surfactants. We were led to the use of Castrol motor oil by the reasoning that gasoline engine lubricating oil contains additives whose purpose is to keep mineral and metal particles in suspension.

[19] Ultraol $50 \mathrm{NF}$ is a mineral oil used in pharmaceutical and cosmetic preparations manufactured by Ultra Chemical, Inc.

[20] C. D. Hendricks et al., Meas. Sci. Technol. 5, 337 (1994).

[21] D. C. Joyce et al., Phys. Rev. Lett. 51, 731 (1983).

[22] M. L. Savage et al., Phys. Lett. B 167, 481 (1986).

[23] I. T. Lee et al., Phys. Rev. D 66, 012002 (2002).

[24] H.-C. Yeh in Aerosol Measurements, edited by K. Willeke and P. A. Baron (Wiley, New York, 1993), pp. 410-417.

[25] S. Fan et al., Rev. Sci. Instrum. 74, 4305 (2003).

[26] E. R. Lee et al., Metrologia 41, S147 (2004). 